\title{
A STUDY AND ANALYSIS OF DIFFERENT EDGE DETECTION TECHNIQUES
}

\author{
Gullanar M. Hadi ${ }^{1}$ and Nassir H. Salman ${ }^{2}$ \\ ${ }^{1}$ Department of Software Engineering, Salahaddin University,Erbil, Iraq \\ gullanarm@yahoo.com \\ ${ }^{2}$ Department of Computer Science, Cihan University, Erbil, Iraq \\ nahu64@yahoo.com
}

\begin{abstract}
In the first study [1], a combination of K-means, watershed segmentation method, and Difference In Strength (DIS) map were used to perform image segmentation and edge detection tasks. We obtained an initial segmentation based on K-means clustering technique. Starting from this, we used two techniques; the first is watershed technique with new merging procedures based on mean intensity value to segment the image regions and to detect their boundaries. The second is edge strength technique to obtain accurate edge maps of our images without using watershed method. In this technique: We solved the problem of undesirable over segmentation results produced by the watershed algorithm, when used directly with raw data images. Also, the edge maps we obtained have no broken lines on entire image. In the 2nd study level set methods are used for the implementation of curve/interface evolution under various forces. In the third study the main idea is to detect regions (objects) boundaries, to isolate and extract individual components from a medical image. This is done using an active contours to detect regions in a given image, based on techniques of curve evolution, Mumford-Shah functional for segmentation and level sets. Once we classified our images into different intensity regions based on Markov Random Field. Then we detect regions whose boundaries are not necessarily defined by gradient by minimize an energy of Mumford-Shah functional for segmentation, where in the level set formulation, the problem becomes a mean-curvature which will stop on the desired boundary. The stopping term does not depend on the gradient of the image as in the classical active contour. The initial curve of level set can be anywere in the image, and interior contours are automatically detected. The final image segmentation is one closed boundary per actual region in the image.
\end{abstract}

\section{KEYWORDS}

Watershed, difference in strength map, K-means, edge detection, image segmentation. Active counters, Level set method, Markov Random Field

Sundarapandian et al. (Eds) : ITCS, SIP, CS \& IT 09, pp. 39-50, 2013. ( C CS \& IT-CSCP 2013

DOI : $10.5121 /$ csit.2013.3105 


\section{INTRODUCTION}

Edges are boundaries between different textures. Edge also can be defined as discontinuities in image intensity from one pixel to another. The edges for an image are always the important characteristics that offer an indication for a higher frequency. Detection of edges for an image may help for image segmentation, data compression, and also help for well matching, such as image reconstruction and so on. In the method of image segmentation, we focus on the idea that edges define boundaries and that regions are contained within these edges. Edge detection refers to the process of identifying and locating sharp discontinuities in an image. There are many methods to make edge detection as follows:

To perform image segmentation and edge detection tasks, there are many methods that incorporate region-growing and edge detection techniques, for example, it is applying edge detection techniques to obtain Difference In Strength (DIS) map then employ region growing techniques to work on the map as in [1] and [2]. In [3], combining both special and intensity information in image segmentation approach based on multi-resolution edge detection, region selection and intensity threshold methods. As in [4], Pappas considered the problem of segmenting images with smooth surfaces, which presents a generalization of the K-means clustering algorithm to include special constraints and to account for local intensity variations in the image. Qixiang Ye et al. [5] have proposed to find main edges meanwhile filter edges within texture regions. They have computed pixel similarity degree around a pixel, have computed a new gradient, and applied a Canny like operator to detect and locate edges. Caragea [6] detects the difference between pairs of pixel around a pixel and uses the highest value from the difference of four pairs of pixels that can be used to form a line through the middle pixel. Al-amri et al. [7] presented methods for edge segmentation of satellite image: they used seven techniques for this category; Sobel operator technique, Prewitt technique, Kiresh technique, Roberts technique, Laplacian technique, Canny technique and Edge Maximization Technique (EMT) and they are compared with one another so as to choose the best technique for edge detection segment image. P. Thakare [8] discussed about some image segmentation techniques like edge based, region based and integrated techniques and explains in brief the edge based techniques and their evaluation. They also focuses on edge based techniques and their evaluation.

\section{LEVEL SET METHOD}

In mathematics, a level set of a real-valued function $f$ of $n$ variables is a set of the form:

$$
\left\{\left(x_{1}, \ldots, x_{n}\right) \mid f\left(x_{1}, \ldots, x_{n}\right)=c\right\}
$$

where $c$ is a constant. That is, it is the set where the function takes on a given constant value. When the number of variables is two, this is a level curve (contour line), if it is three (in 3-D ; Surface (Interface) evolution), this is a level surface, and for higher values of $n$ the level set is a level hyper surface. So level set methods are used for the implementation of curve/interface evolution under various forces . In this method many modified functions were used: [4] and [9] 
a) Function evolve2D () was used which is a high level function that takes an input, evolves it $\mathrm{N}$ iterations and returns the result. b) Function contour $(\mathrm{Z})$ is a contour plot of matrix $\mathrm{Z}$ treating the values in $\mathrm{Z}$ as heights above a plane. A contour plot is the level curves of $\mathrm{Z}$ for some values $\mathrm{V}$. The values $\mathrm{V}$ are chosen automatically. The contours are normally colored based on the current color map. c). Function imcontour; where imcontour (I) was used to create contour plot of image data and to draw a contour plot of the intensity image I. We found that 50 iterations are very good for some images, and the evolution type depend upon the parameters values in function Phi[4][9]:see Figure (6)

phi $=$ evolve $2 \mathrm{D}(\mathrm{phi}, \mathrm{dx}, \mathrm{dy}, 0.5,25,[],[], 0,[], 0,[],[], 1, \mathrm{~b})$; also the processing time depend upon iteration numbers.

\section{Edge Strength Merging Process}

Two edge strengths gradient values $\left(\mathrm{T}_{1}, \mathrm{~T}_{2}\right)$ were used in one subroutine, $\mathrm{T}_{1}$ is less than $\mathrm{T}_{2}$. For example if we choose $T_{1}=1$ and if the Edge strength as in equation (2) is less than 1, we get merging of every two adjacent regions because the watershed algorithm [10] we used based on immersion procedure and in this procedure it looks to the topographic surface. It means we related intensity values as an altitude (height) and we got merging results by comparing the gradient values of the edge points (pixels) between the two regions and the region itself. If the points have low gradient values, that means the merging was done and the region becomes large. So; in this procedure it is very important and useful the choosing values of $T_{1}, T_{2}$ in our merging process. See the results in Figure 3.

$$
\text { Edge strength }=\frac{\sum_{p \in \text { Edge }} \text { Gradient }(p)}{N}
$$

Where Gradient (p) represents edge points gradient values which come from the gradient image step for all pixels (p) on the edge between every two regions, and $\mathrm{N}$ are the number of edge pixels.

\subsection{Edge Strength Merging Process results}

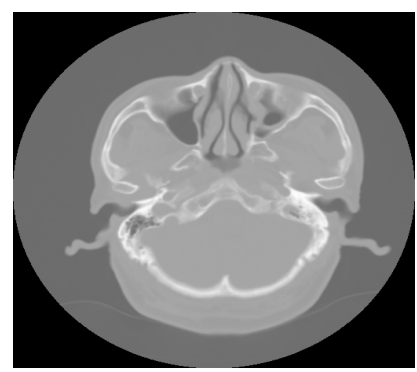

(a) Original image of Brain image (512x512).

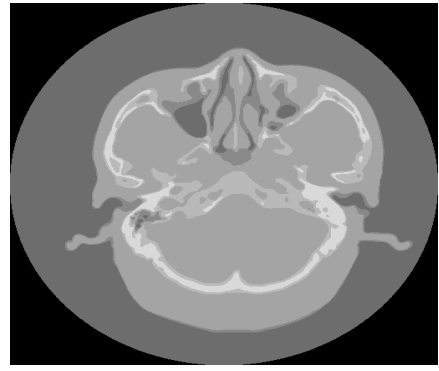

(b) Seg. image into 6 regions by k-means method. 


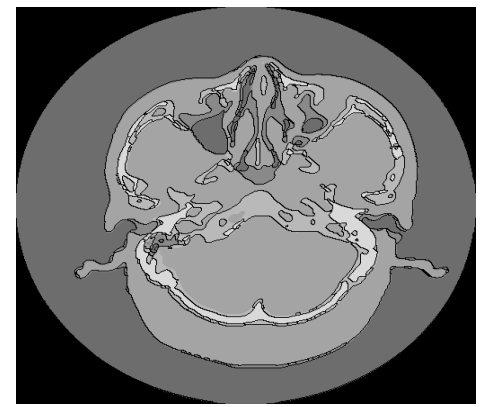

(c) Then segmented image with edges (region map) by watershed algorithm.

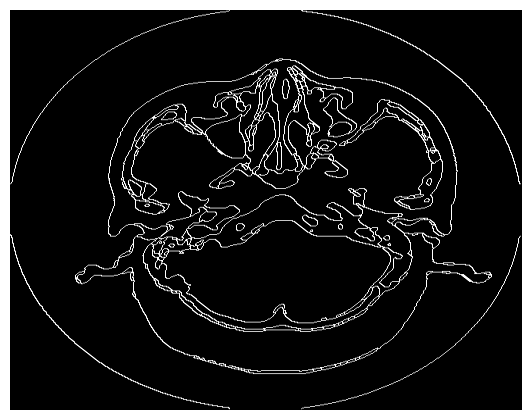

(d) An accurate edge map after watershed $\&$ merge process by mean value.

Figure 1. The results of K-means method, watershed algorithm, and merging techniques. $[\mathrm{cpu}=27.835 \mathrm{~s}$. [20.936 $s$ for K-means. $6.229 s$ for watershed and merging. And $0.670 s$ for two edge strength].

\section{Difference in Strength TechniQue Results}

The DIS for each pixel was calculated using equation (3) [11]. And after processing all the input pixels, the DIS map was obtained. In DIS map, the larger the DIS value is, the more the pixel is likely located at the edge. At this step, a $3 \times 3$ window runs pixel by pixel on the input image. When the window runs over the bolder of the input image, pixels outside the bolder are given the gray level of the input nearest to it. The DIS for the center pixel as in Figure 2, for example, was calculated as in equation (3) [11].

$$
\begin{aligned}
& \left|Z_{1}-Z_{3}\right|+\left|Z_{1}-Z_{5}\right|+\left|Z_{1}-Z_{6}\right|+\left|Z_{1}-Z_{7}\right|+\left|Z_{1}-Z_{8}\right| \\
& +\left|Z_{2}-Z_{4}\right|+\left|Z_{2}-Z_{5}\right|+\left|Z_{2}-Z_{6}\right|+\left|Z_{2}-Z_{7}\right|+\left|Z_{2}-Z_{8}\right| \ldots \ldots \ldots \ldots \ldots . .(3) \\
& \left|Z_{3}-Z_{4}\right|+\left|Z_{3}-Z_{6}\right|+\left|Z_{3}-Z_{7}\right|+\left|Z_{3}-Z_{8}\right|+\left|Z_{4}-Z_{5}\right| \\
& +\left|Z_{4}-Z_{7}\right|+\left|Z_{4}-Z_{8}\right|+\left|Z_{5}-Z_{6}\right|+\left|Z_{5}-Z_{7}\right|+\left|Z_{6}-Z_{8}\right|
\end{aligned}
$$



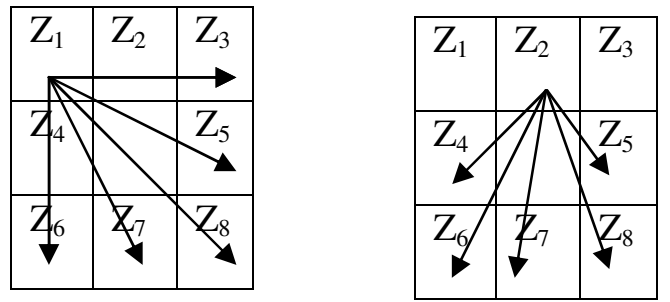

Figure 2. The DIS detecting windows.

Examples of DIS maps are shown in Figure 3-b. One can expect that the values of DIS should be small in the smooth regions obtained by k-means. The greater DIS value represents that the pertaining pixel is on the area that changes severely in gray levels. With the DIS map one can check with the result of image segmentation based on K-means. It is clear that the DIS map consists of all edge information about the input image even on the smooth regions.

Since the DIS of the smooth region is small (weak edge), one can use a threshold $T$ to eliminate false edges and thus obtain larger regions. In this case, the DIS map provides the complete edge (strong and weak) information about the image. By exploiting these information, one can accurately locate the contour of an object. Now to find the effect of DIS, we used multithreshoding edge detection; first we calculated DIS for each pixel in the image then we calculated the mean value of DIS for the whole image. From the mean value we thresholded our image by different $\%$ of mean DIS. The threshold for discarding weak edges is set to the mean of $D I S$ as in Figure 5 (d through $\mathrm{g}$ ).

The threshold used for connected edges is set to the $50 \%$ of mean DIS. So, using multi-threshold is important to eliminate false edges and thus obtain larger regions as in Figure 3 (d through $\mathrm{g}$ ). The region map without threshold is shown in Figure 3-c. As we can see from the Figure 3 (d-g) compare with the Figure 3-c, the concept that an object should have a closed contour help us to eliminate redundant edge pixels and connect the broken contour by using multi-threshold based on different values of mean DIS of the whole image under study. But if we take k-means and then $D I S$ with $25 \%$ of mean DIS, we will get all the edges of our images as in the Figure 4 below and we don't need to use watershed technique. 


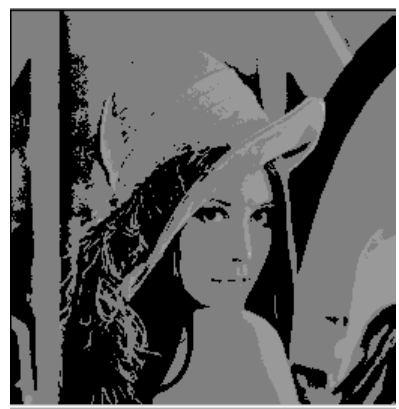

(a) Original image.

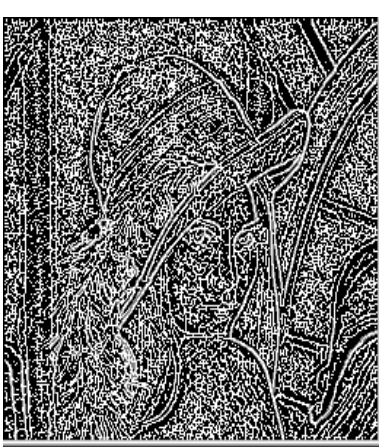

(c) Without threshold

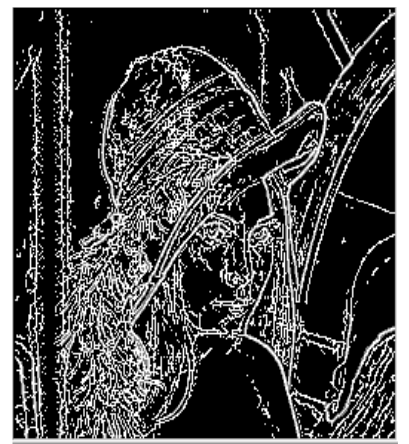

(e) $\mathrm{T} 50 \%$ of $\mathrm{DIS}$ mean.

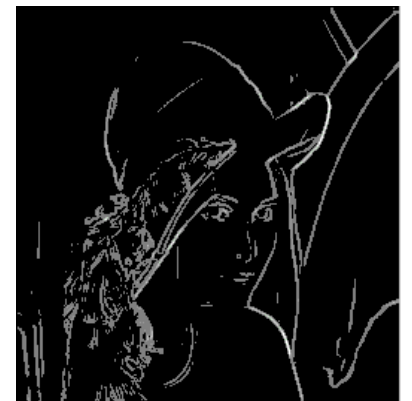

(b) DIS map of image (a).

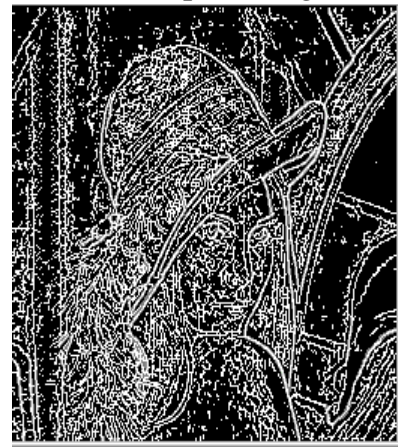

(d) Threshold $25 \%$ of DIS mean.

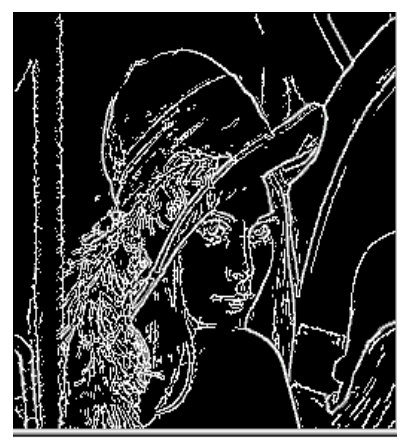

(f) $\mathrm{T} 100 \%$ of $\mathrm{DIS}$.

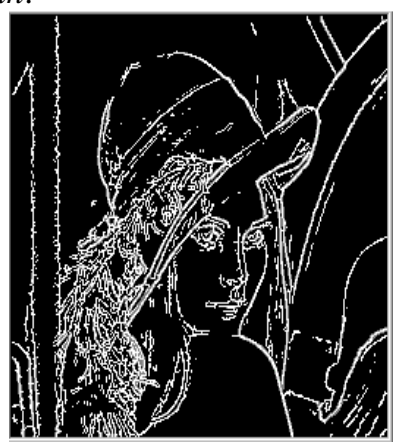

(g) T $120 \%$ of DIS mean.

Figure 3. DIS map of image and multithreshoding of DIS mean edge detection results. 


\subsection{Other DIS Method Results}

The experimental results are shown in Figures $(1,3)$ above and Figure (4) below. Medical images as brain images are simple pattern images with the size of $\{156 \times 156\}$ and 256 gray levels images and other images to test our segmentation and edge detection methods. We obtained output images consist of all edge information and regions about the input image. The region maps are shown in Figure1-c. As can be seen from the edge maps Figure 1-d, that there are no broken lines on the whole image regions. The output image was displayed as an edge map as in Figure 1d, Figure 3(e through g); and Figure 4-c.

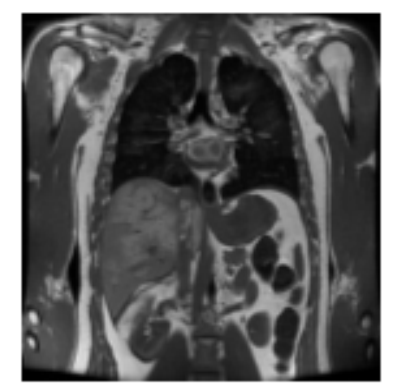

(a) Original image.

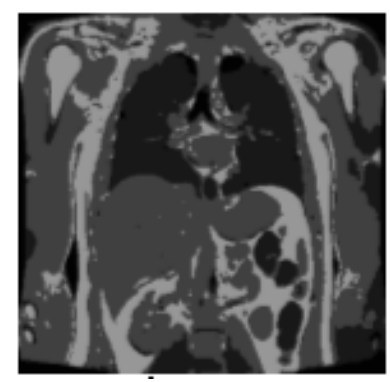

(b) After K-means process.

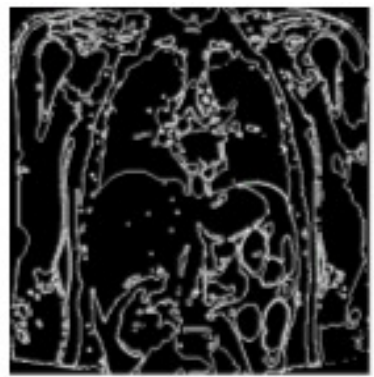

(c) Using threshold $25 \%$ of mean DIS.

Figure 4. Edge map using K-means process and thresholding $5 \%$ of mean DIS.

\section{ACtive Contour Results Based on Level SeT}

The basic idea in active contour models or snakes is to evolve a curve, subject to constraints from a given image, in order to detect objects in that image. For instance, starting with a curve around the object to be detected, the curve moves toward its interior normal and has to stop on the boundary of the object. In the classical snakes and active contour models (see [12], [13],[14], [15]), an edge-detector is used, depending on the gradient of the image $\boldsymbol{u}_{\mathbf{0}}$, to stop the evolving curve on the boundary of the desired object.during our process, we used initial segmented images (different intensity regions) based on Markov Random Field to superimpose the region boundary and to extract the bounded region (segmented map) in our image as in Figure. 5 (b \& c) as an example. 


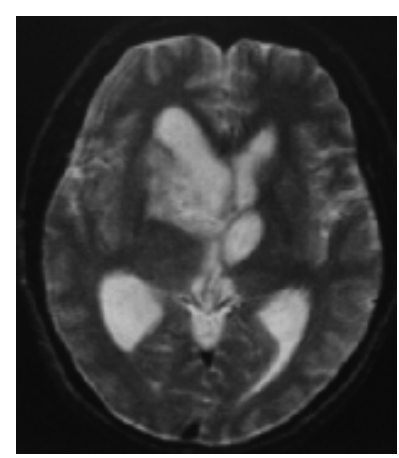

Original image

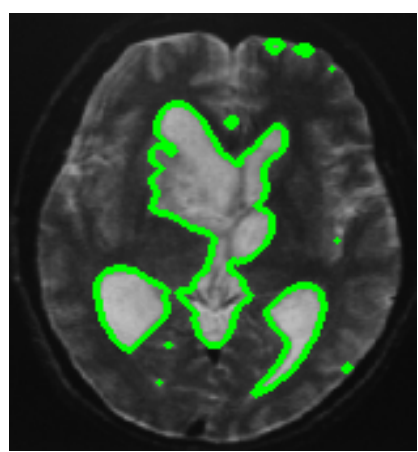

(b) Segmented image by

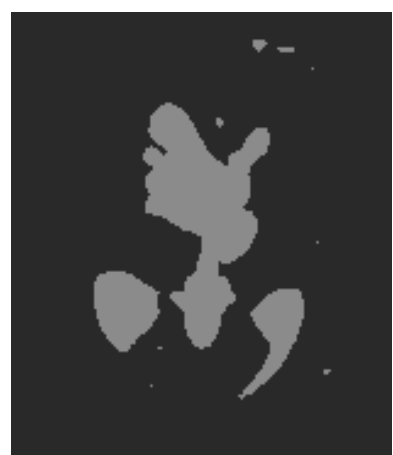

(c) Segmented map of

M-Shah GAC method bounded area in (b) (extracted region )
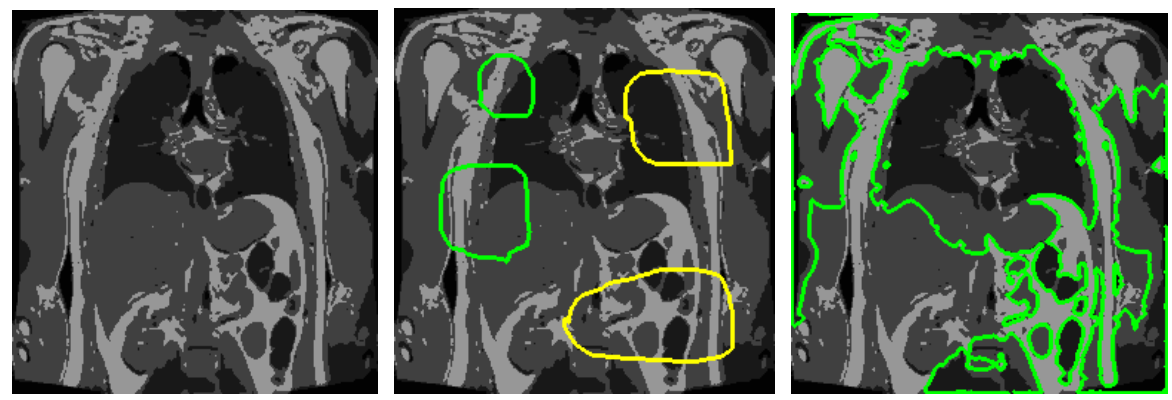

(d) Abdomen image after

(e) Stepl initial curves

(f) Step 2

MRF method

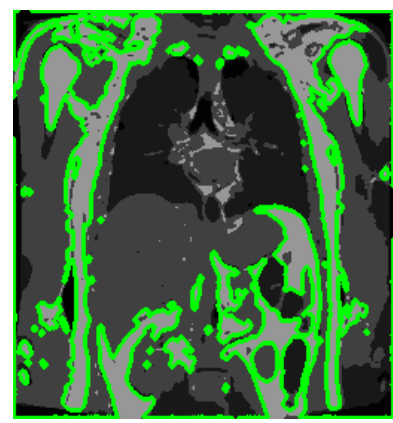

(g) Step3

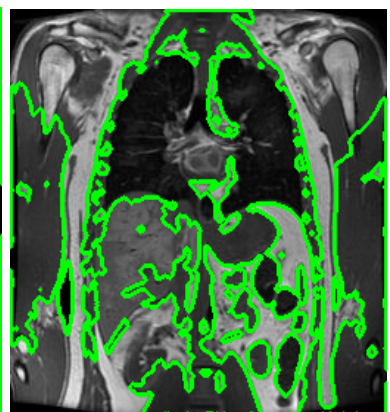

(h) Semi final results

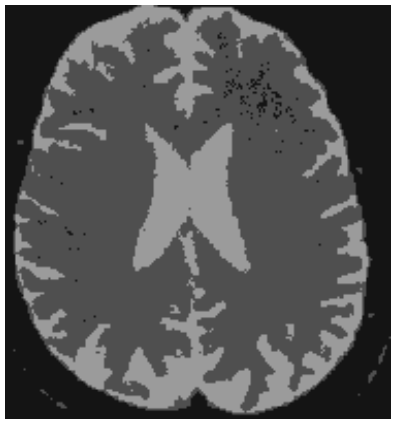

(i) Brain image after $M R F$ 


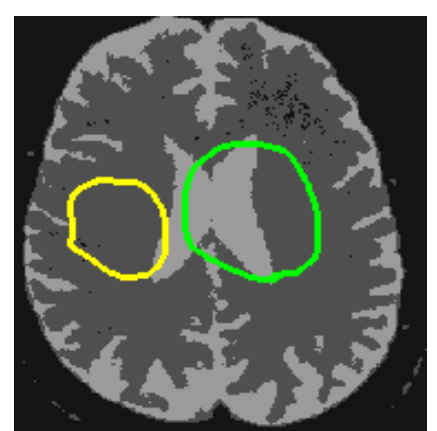

(j) Initial curves

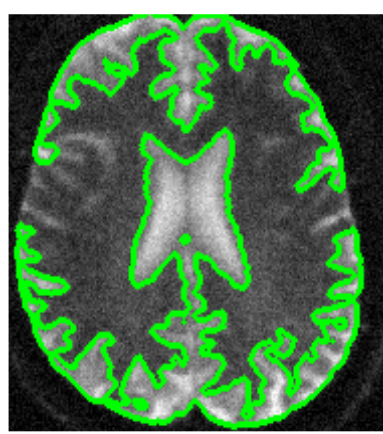

(k) Final results

Figure . 5 Segmentation results by Mumford-Shah Geodesic Active Contours (GAC)

So we superimposed the edge of the different regions in the image using Mumford-Shah method after we chose few closed curves represent different intensity area in our image. For example as shown in Figure.5 (e\&j). So our results accuracy depend on, if the results of MRF is accurate then the regions boundaries are in correct position as shown from the figures above. Also in this method; if we want to choose any region in the image and to define its edge, we can do all that. Then we can calculate some region information such as the area of that region, region map and contour length clearly. We can use different kind of images to extract different features (roads, rivers, agricultural areas ...etc) as in remote sensing images.

\section{Levelset Methods Results Using Matlab as IN FIG(6)}
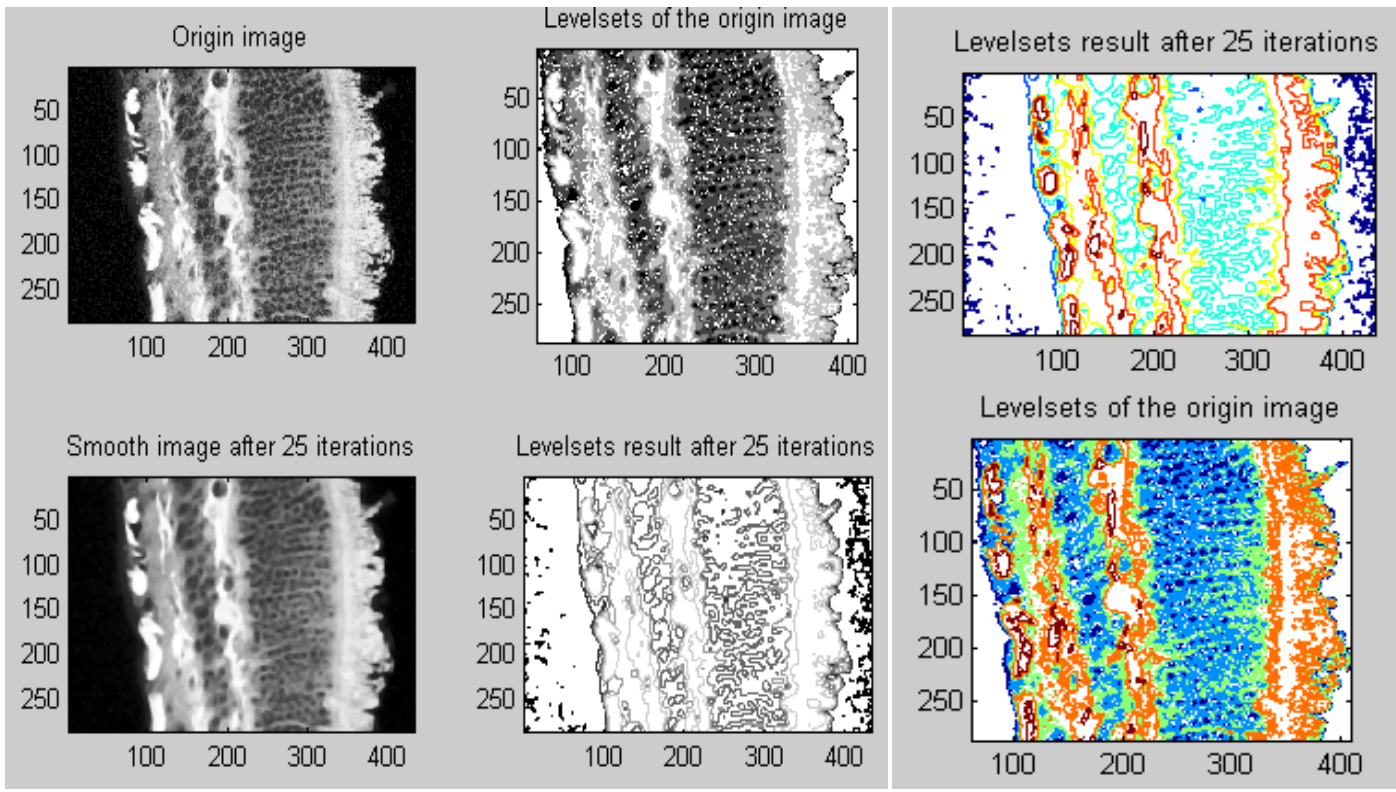

Figure 6. The final results of levelset method to brain image and elapsed time of processing

$(28.5 \mathrm{sec})$ 


\section{CONCLUSION}

In DIS method:, the segmentation regions and their boundaries were defined well and all of the boundaries are accurately located at the true edge as shown clearly from Figure1-(c, d), Figure 3g, and Figure 6-c. And if we take k-means first and then DIS with $25 \%$ of mean DIS, we will get all the edges of our images as shown in the Figure 4 above, so we need'nt to use watershed technique.

Also we concluded that using multi-threshold is important to eliminate false edges and thus obtain larger regions, the DIS map consists of all edge information about the input image even on the smooth regions, and the combination of k-means, watershed segmentation method, DIS map are good techniques to perform image segmentation and edge detection tasks, where the final segmentation results are one closed boundary per actual region of the image under study, and the two edge strengths gradient values $\left(\mathrm{T}_{1}, \mathrm{~T}_{2}\right), \mathrm{T}_{1}$ is less than $\mathrm{T}_{2}$, are very sensitive to get good results. Where the incorrect choosing of these values gives us uncorrected image segmentation and edge detection results and this is a disadvantage. So we will develop this work in future with automatically determined the threshold values.

Finally, the disadvantages of these techniques are depending mainly on k-means results, where if the clustering procedure doesn't implement correctly, the results are incorrect by the other techniques we used. However, in this paper we solved the problem of undesirable oversegmentation results produced by the watershed algorithm, also the edge maps we obtained have no broken lines on entire image.

In levelset method: we conclude that the levelset technique and extraction an object methods give us very accurate and clear results. We found that 50 iterations are very good for some images, and the evolution type depend upon the parameters values in function Phi[4,8]:see Figure (6)

phi $=$ evolve2D $(\mathrm{phi}, \mathrm{dx}, \mathrm{dy}, 0.5,25,[],[], 0,[], 0,[],[], 1, \mathrm{~b})$; also the processing time depend upon iteration numbers. In the other study using an active contours based on techniques of curve evolution, Mumford-Shah functional for segmentation and level sets is a good and accurate method to detect object(region) boundaries, to isolate and extract individual components from our image. It is possible to detect objects whose boundaries are not necessarily defined by gradient by minimize an energy of Mumford-Shah functional for segmentation which can be seen as a particular case of the minimal partition problem where the stopping term does not depend on the gradient of the image, as in the classical active contour and the initial curve of level set can be anywhere in the image. This help us to obtain the final image segmentation is one closed boundary per actual region in the image where the segmentation problem involves finding the closed curve $\mathrm{C}$ that lies along the boundary of the object of interest in the image. Then it is easy to calculate the region area and the boundary length. The level set approach allows the evolving front which can extract the boundaries of particularly intricate contours. Also we can use this method with different kind of images (e.g., medical images and remote sensing images) to detect object boundaries, to isolate and extract individual components (as a segmented maps) as shown as an example in Figure 5 (c).finally Edge detection refers to the process of identifying 
and locating sharp discontinuities in an image. The discontinuities are abrupt changes in pixel intensity which characterize boundaries of objects in a scene.

\section{References}

[1] Salman N and Liu C. Q.,(2003) “Image Segmentation and Edge Detection Based on Watershed Techniques", International Journal of Computers and Applications, Vol. 25, No. 4, pp. 258-263.

[2] Yu, Yi-Wei, Wang, Jung-Hua.,(1999) Proc. of the IEEE international conference on Systems, Man and Cybernetics (SMC), 6 :P-798

[3] Chowdhury, Mahbubul Islam; Robinson, John A.,(2000) IEEE Proc. of Canadian Conference on Electrical and Computer Engineering, 1: P-312

[4] Tang, H. , Wu, E. X., et al.(2000) Computerized Medical Imaging and Graphics,Vol. 24,No. 6,P 349

[5] Qixiang, Ye, Wen, G., Weiqquiang, W. (2003) "A New Texture Insensitive Edge Detection Method",Institute of Computing Technology, Chinese Academy of Sciences ,China, ICICS-PCM 2003, 15-18 Dec 2003, Singapore.

[6] Caragea S. 2008. Fondater, Administrator and Chief Editor, IntelliProject, "Difference Edge Detection“, Licensed under IntelliProject open License, Romania, http://www.intelliproject.net

[7] S. S. Al-amri, N. V. Kalyankar and S. D. Khamitkar,( 2010 )"Image Segmentation by using Edge Detection",International Journal on Computer Science and Engineering (IJCSE),Vol. 02,No.03, pp. 804-807.

[8] Puman Thakare,(2011) "A study of image segmentation and edge detection techniques", International Journal of Computer Science and Engineering (IJCSE), Vol. 3, No. 2, Feb 2011.

[9] BarisSumengen, (2005)A Matlab toolbox implementing Level Set Methods., vision research lab at UC Santa Barbara.

[10] Matlab the language of technical computing, version 7.6.0.324(R2008a).

[11] Vincent L. and Soille P. (1991) "Watershed in Digital Space: An Efficient Algorithm Based on Immersion Simulations," IEEE Transactions on Pattern Analysis and Machine Intelligence,Vol. 13, No. 6, pp. 583-593

[12] Yu Y. and Wang J., (1999)“Image Segmentation Based on Region Growing and Edge Detection,” in Proceedings of the 6th IEEE International Conference on Systems, Man and Cybernetics, Tokyo, Vol.6., pp. 798-803.

[13] M. Kass, A. Witkin, and D. Terzopoulos,(1988) "Snakes: Active contourmodels," Int. J.Comput.Vis., Vol. 1, pp. 321-331, 1988.

[14] V. Caselles, F. Catté, T. Coll, and F. Dibos,(1993) "A geometric model for active contours in image processing,” Numer. Math., vol. 66, pp. 1-31, 1993.

[15] R. Malladi, J. A. Sethian, and B. C. Vemuri,(1993) "A topology independent shape modeling scheme," in Proc. SPIE Conf. Geometric Methods ComputerVision II, Vol. 2031, San Diego,CA, pp. $246-258$. 
[16] V. Caselles, R. Kimmel, and G. Sapiro, "On geodesic active contours," Int. J. Comput. Vis.,Vol. 22, No. 1, pp. 61-79, 1997.

\section{Author}

Gullanar M. Hadi, born in 1964. She received her B.Sc. and M.Sc. degrees from Collage of Science, Al-Mustansyriah University, Baghdad -IRAQ in 1985 and 1989 respectively. She received his $\mathrm{PhD}$ degree in Opto-Electronics engineering from Shanghai Jiao Tong University-China in 2004. Her research interests include image processing and nano-materials. From 1985-2006, she worked at AlMustansyriah University, collage of Science, Baghdad -IRAQ, from 2006-2009 she worked as a lecturer in several Jordanian University, from 2009 to present she was lecturer in Software Engineering dept. , Salahaddin University, Erbil-Iraq.

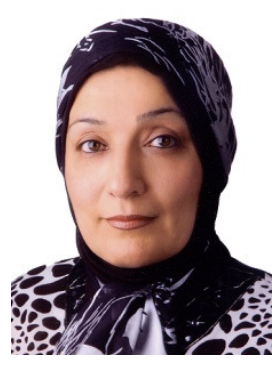

Corresponding author: SALMAN, N. H, born in 1960. He received his B.Sc. and M.Sc. degrees from Al-Mustansyriah University / Collage of Science in 1983 and 1989 respectively. He received his PhD degree in Image Processing and Pattern Recognition from Shanghai Jiao Tong University-China in 2002. His research interests include remote sensing, image processing and image analysis based on image segmentation and edge detection techniques. Also he is interesting in computer programming languages, Matlab programming. From 1982-1998, he worked at Space Research Center -Remote Sensing Dept. in Baghdad -IRAQ, also he was an assistant professor (from 2002) in the Dept. of Computer Science-Zarqa

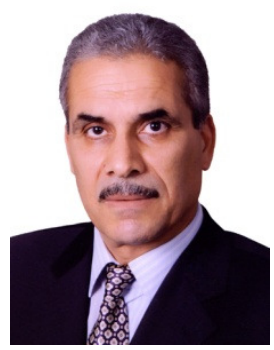
Private University-Jordan. Now he is a head of Computer Science dept .Cihan University, Erb 